\title{
Re: The Use of Enoxaparin to Prevent Venous Thromboembolism in Patients Undergoing Radical Retropubic Prostatectomy: Feasibility and Utility
}

\author{
Kogenta Nakamura, Ali Kasraeian, Saif Yacoub, John Pendleton, Satoshi Anai, Charles J. Rosser \\ Division of Urology, University of Florida, Jacksonville, and Department of Urology, University of \\ Florida, Gainesville, Florida, USA
}

Int Braz J Urol, 33: 347-354, 2007

To the Editor:

It is very interesting to me that the use of an anticoagulant such as heparin is "standard" prophylaxis after an open radical prostatectomy (RP) in much of Western Europe but not in the US. I do not know if it is used in conjunction with a laparoscopic RP but it would seem that there would be little difference.

There is no argument that venous thromboembolism (VTE) is the most important nonsurgical complication following major urologic surgery and this would include RP. The rate of VTE has been steadily declining over the past two decades thanks to improved techniques during surgery and thus less blood loss, i.e. less risk of hypotension, a lower operative time, earlier mobilization, and the use of VTE prophylaxis. Despite these advances, the incidence of symptomatic VTE ranges between 1 and $5 \%$. Pulmonary embolus, although quite uncommon, is the most often cited cause of post RP death $(<1$ in 500).

Indeed patients undergoing a RP have known risk factors for a VTE, such as older age, pelvic surgery, node dissection, cancer. Thus the consensus for some method to reduce the risk of a VTE. There are very few recent prospective trials, which compare different methods for VTE prophylaxis in urologic surgery. The three commonly used approaches to VTE prophylaxis are graduated compression stockings (GCS), intermittent pneumatic compression devices (IPCD), and pharmacologic therapy, i.e. one of the heparin products.

An outstanding review of VTE prophylaxis was published in 2004 (1). The recommendation for urologic surgery and specifically major open procedures such as RP was routine prophylaxis with low dose unfractionated heparin two or three times daily. Acceptable alternatives include IPCD and /or GCS or low molecular weight heparin. Thus, we have a choice. No perfect answer.

What do I do? For the past 15 years our anesthesia team and I have used a protocol which consists of a long acting spinal supplemented by general anesthesia(2). Patients are positioned in the supine flexed position with the kidney rest raised. IPCD are placed when the patient enters the operating room and are maintained during surgery and until the next morning when the patient is out of bed and ambulating. Ninety percent of patients are discharged the day after surgery without additional VTE prophylaxis.

We reported our incidence of VTE in 1,364 consecutive RP in 2005 (3). There were three VTE events $(0.21 \%)$ in lower $(n=2)$ or upper $(n=1)$ extremities. No patient had a clinical pulmonary embolus. The only postoperative death was from a myocardial infarction. Since that publication, there have been no additional clinical VTE.

The use of a spinal anesthetic may be an important component to our low incidence of VTE. Prospective trials have convincingly demonstrated that patients receiving a spinal or epidural anesthetic with or without a concurrent general component have a significantly reduced chance of a VTE (4). The precise mechanism is not clear but less stasis in the lower extremities or lower blood loss may be factors. The long acting spinal actually encourages early 
ambulation since the patients have less postoperative pain.

The article by Nakamura et al. asks what to do when patients do not comply with the IPCD. My suggestion would be to emphasize to the nurses and the patient the importance of the devise and in addition use a spinal anesthetic. Our patients remove the devices the morning after surgery and begin ambulation.

\section{References}

1. Geerts WH, Pineo GF, Heit JA, Bergqvist D, Lassen MR, Colwell CW, et al: Prevention of venous thromboembolism: the seventh ACCP conference on antithrombotic and thrombolytic therapy. Chest. 2004; 126: 338S-400S.

2. Sved P, Nieder A, Manoharan M, Gomez P, Meinbach DS, Kim SS, et al: Evaluation of analgesic requirements and postoperative recovery after radical retropubic prostatectomy utilizing long acting spinal anesthesia. Urology. 2005; 65: 509-12.

3. Koya MP, Manoharan M, Kim S, Soloway MS: Venous thromboembolism in radical prostatectomy: is heparanoid prophylaxis warranted? BJU Int. 2005; 96: 1019-21.

4. Rodgers A, Walker N, Schug S, McKee A, Kehlet H, van Zundert A, et al: Reduction of postoperative mortality and morbidity with epidural or spinal anesthesia: results from overview of randomised trials. BMJ. 2000; 321: 1493.

Dr. Mark S. Soloway

Professor \& Chair, Department of Urology Miller School of Medicine, University of Miami

Miami, Florida, USA

E-mail: MSoloway@med.miami.edu

\title{
Re: Inflammatory Atrophy on Prostate Needle Biopsies: Is There Topographic Relationship to Cancer?
}

\author{
Athanase Billis, Leandro L.L.Freitas, Luis A. Magna, Ubirajara Ferreira \\ Departments of Anatomic Pathology (AB,LLLF), Genetics and Biostatistics (LAM), and Urology(UF), \\ School of Medicine, State University of Campinas (Unicamp), Campinas, São Paulo, Brazil
}

Int Braz J Urol 2007;33:355-63

To the Editor:

The editorial comments of our paper by Dr.H.Samaratunga, Dr. Rodolfo Montironi, and Dr. Liang Cheng were very informative on a lesion that is one of the most frequent mimics of prostatic adenocarcinoma. It occurs most frequently in the posterior lobe or peripheral zone (1-3) and gained im- portance with the increasing use of needle biopsies for the detection of prostatic carcinoma (4). Moore (1), in 1936, was one of the first authors to describe prostatic atrophy in a systematic autopsy study. He found that there was a strong correlation with age and, according to his study, prostatic atrophy is initi- 